\title{
High-Dose Erythropoietin for Asphyxia and Encephalopathy (HEAL): A Randomized Controlled Trial - Background, Aims, and Study Protocol
}

\author{
Sandra E. Juul ${ }^{\mathrm{a}}$ Bryan A. Comstock ${ }^{\mathrm{b}}$ Patrick J. Heagerty ${ }^{\mathrm{b}}$ Dennis E. Mayock ${ }^{\mathrm{a}}$ \\ Amy M. Goodman ${ }^{c}$ Stephanie Hauge ${ }^{a}$ Fernando Gonzalez ${ }^{c}$ Yvonne W. Wu ${ }^{c}$ \\ a Department of Pediatrics, Division of Neonatology, University of Washington, Seattle, WA, USA; ${ }^{b}$ Department of \\ Biostatistics, University of Washington, Seattle, WA, USA; ' Department of Neurology, Division of Child Neurology, \\ University of California, San Francisco, CA, USA
}

\section{Keywords}

Hypoxia-ischemia · Neonatal encephalopathy · Therapeutic hypothermia $\cdot$ Neuroprotection

\begin{abstract}
Background: Hypoxic-ischemic encephalopathy (HIE) remains an important cause of neonatal death and frequently leads to significant long-term disability in survivors. Therapeutic hypothermia, while beneficial, still leaves many treated infants with lifelong disabilities. Adjunctive therapies are needed, and erythropoietin (Epo) has the potential to provide additional neuroprotection. Objectives: The aim of this study was to review the current incidence, mechanism of injury, and sequelae of $\mathrm{HIE}$, and to describe a new phase III randomized, placebo-controlled trial of Epo neuroprotection in term and near-term infants with moderate to severe HIE treated with therapeutic hypothermia. Methods: This article presents an overview of HIE, neuroprotective functions of Epo, and the design of a double-blind, placebo-controlled, multicenter trial of high-dose Epo administration, enrolling 500 neonates $\geq 36$ weeks of gestation with moderate or severe HIE diagnosed by clinical criteria. Results and Conclu-
\end{abstract}

(c) 2018 S. Karger AG, Basel

E-Mail karger@karger.com www.karger.com/neo sions: Epo has robust neuroprotective effects in preclinical studies, and phase I/II trials suggest that multiple high doses of Epo may provide neuroprotection against brain injury in term infants. The High Dose Erythropoietin for Asphyxia and Encephalopathy (HEAL) Trial will evaluate whether highdose Epo reduces the combined outcome of death or neurodevelopmental disability when given in conjunction with hypothermia to newborns with moderate/severe HIE.

(c) 2018 S. Karger AG, Basel

\section{Introduction}

Perinatal asphyxia, caused by a lack of oxygen and perfusion to the brain and other vital organs, globally contributed to an estimated $11 \%$ of deaths among children under 5 years of age, and $22 \%$ of deaths in the first month of life in 2013 [1]. Risk of perinatal asphyxia or hypoxicischemic encephalopathy (HIE) varies widely by country, ranging from 2-4 per 1,000 live births in high-income

ClinicalTrials.gov Identifier: NCT No. 02811263, IND 102,138.
Sandra Juul, MD, PhD

Department of Pediatrics, Division of Neonatology, University of Washington Box 356320

Seattle, WA 98195 (USA)

E-Mail sjuul@uw.edu 
countries up to 26 per 1,000 live births in low/middleincome countries [2]. Therapeutic hypothermia (TH) is currently the only proven treatment for HIE, and is the standard of care in high-income countries [3]. While therapeutic hypothermia is clearly beneficial (the number needed to treat is 8) [3], neonates with moderate/severe HIE treated with therapeutic hypothermia still experienced unacceptably high complications: mortality $28 \%$ (range 24-38); cognitive impairment 24\% (range 21-25); cerebral palsy (CP) 22\% (range 13-28); epilepsy $19 \%$ (range 15-24); cortical visual impairment 6\% (range 1-10), with combined death or moderate/severe disability $48 \%$ (range 44-53) [3]. Adjunctive therapies to further improve outcomes are desperately needed. In high-income countries, experimental therapies must be tested in conjunction with the standard of care.

Erythropoietin (Epo) has neuroprotective and neuroregenerative effects, which are the net effects of the anti-inflammatory, anti-excitotoxic, anti-oxidant, and anti-apoptotic effects on neurons and oligodendrocytes. Furthermore, Epo promotes neurogenesis, oligodendrogenesis, and angiogenesis, which are essential for normal neurodevelopment and injury repair. These beneficial effects of Epo have been well documented in experimental models of neonatal brain injury $[4,5]$, with emerging clinical data also showing benefit [6-8]. The safety profile of high-dose Epo in neonates is also reassuring [9]. Epo pharmacokinetics in HIE and hypothermia have been well defined [10,11]. Given the compelling preclinical data, the suggestive findings from human trials, favorable safety and pharmacokinetic data, and the unacceptable rate of adverse long-term neurologic outcomes in HIE, we are conducting a randomized, placebo-controlled, multicenter, phase III clinical trial in 500 term/near-term infants with moderate/severe HIE to determine whether multiple doses of high-dose Epo reduce the rate of death or neurodevelopmental disability, and reduce the severity of brain injury on neonatal MRI and MRS (the "HighDose Erythropoietin for Asphyxia and Encephalopathy" - HEAL Trial, NCT No. 02811263).

\section{Pathophysiology of HIE}

Lack of both oxygen (hypoxia) and perfusion (ischemia) must be present for a significant period to result in clinically significant HIE. Large animal models suggest occlusion of umbilical cord blood flow for $18-25 \mathrm{~min}$ is sufficient to cause moderate/severe brain injury [12]. The initial insult results in primary energy failure with im- pairment of mitochondrial respiration, anaerobic metabolism, lactic acidosis, failure of cell membrane pumps leading to an influx of sodium and calcium, cell swelling, and death [13]. A cascade of inflammatory events follows with an increased release of pro-apoptotic proteins and subsequent apoptotic cell death. Injury progression continues with ongoing inflammation, impaired neurogenesis, and alteration in synaptogenesis and axonal growth. The mechanism of cell death changes from early necrosis, to later apoptosis with a continuum of phenotypes emerging over time (the apoptosis-necrosis continuum) [14]. The final phase is regeneration and repair. Key targets for neuroprotection might include early anti-oxidant therapy, interruption of acute inflammation and apoptotic cell death, and finally, the stimulation of repair mechanisms.

\section{Epo Neuroprotection}

Epo is unique as a potential neuroprotective agent in that it has acute effects (anti-inflammatory, anti-excitotoxic, anti-oxidant, and anti-apoptotic) and regenerative effects (neurogenesis, angiogenesis, and oligodendrogenesis), essential for repair of injury and normal neurodevelopment $[4,5]$. Epo functions by binding to its homodimeric cell surface receptor (Epo-R). Epo-Rs are expressed by a variety of cell types in the brain, including neuronal progenitor cells, subsets of mature neurons, astrocytes, oligodendrocytes, microglia, and vascular endothelial cells $[4,5]$. Epo and Epo-R expression in the brain is high during fetal development but declines rapidly after birth. During hypoxia-ischemia, Epo-R expression in neurons, astrocytes, and microglia is upregulated [15]. If the insult is of sufficient duration, increased Epo expression follows, mediated via hypoxia-inducible factor-1 $\alpha$. In the absence of Epo-Epo-R binding, cells are predisposed to apoptosis, while in the presence of Epo, cells are preserved [15]. The established functions of Epo create an important rationale for providing exogenous Epo administration to infants with HIE, given that brain injury can occur after brief but catastrophic insults such as placental abruption or cord accidents, and such events are insufficient to stimulate an endogenous increase in Epo synthesis.

In animal models and stroke studies, Epo has been shown to reduce neuronal loss, learning impairment, and $\mathrm{CP}$ following brain injury. These effects are dose dependent, with multiple doses being more effective than a single dose $[16,17]$. Even when initiated as late as 1 week after injury in neonatal rodents, there is evidence of improved behavioral outcomes, enhanced neurogenesis, increased axonal sprouting, and reduced white matter injury [17]. 
Table 1. Inclusion and exclusion criteria

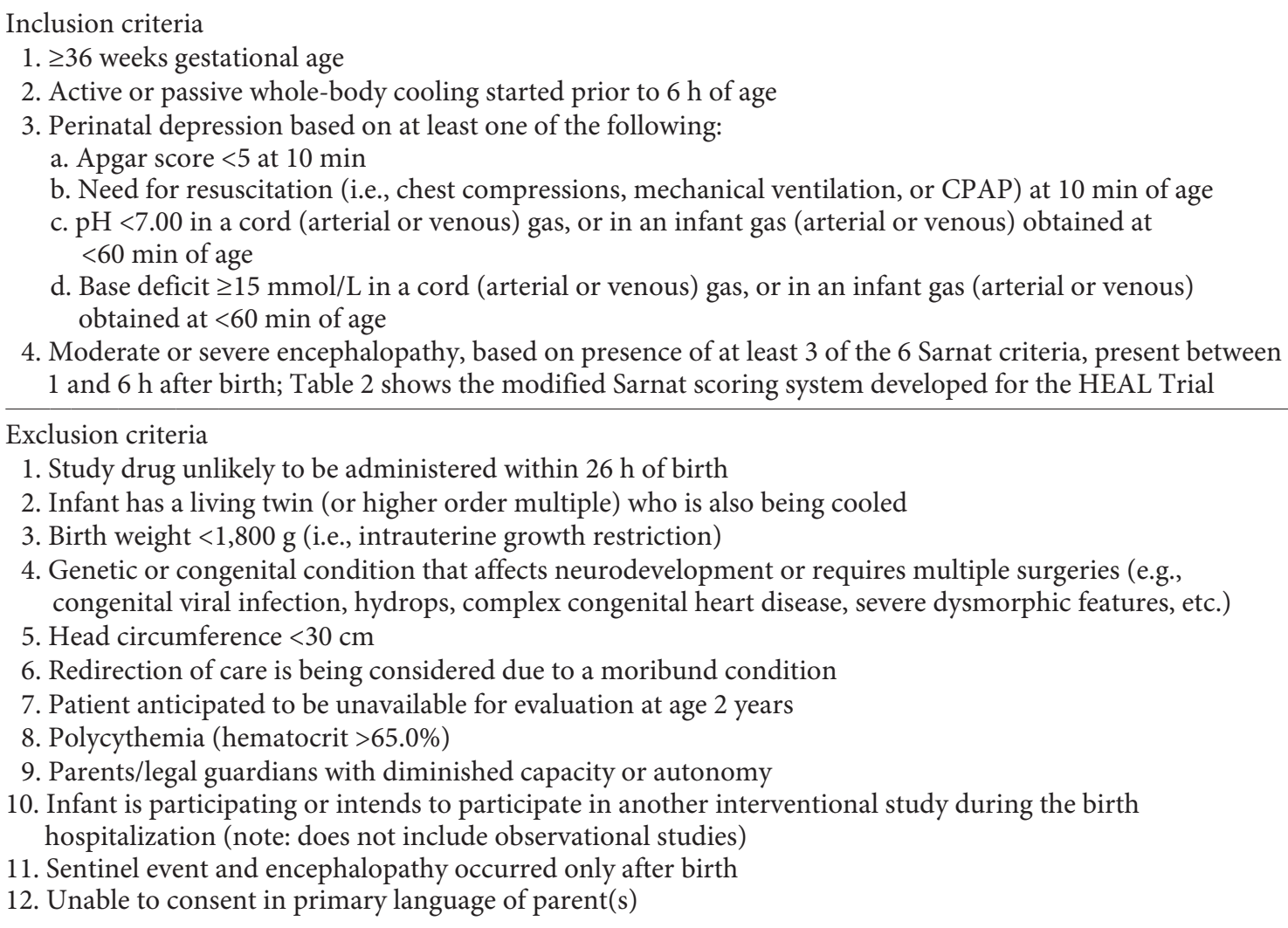

\section{Clinical Studies of Epo Neuroprotection for HIE in the Setting of Therapeutic Hypothermia}

In a phase I open label dose escalation study of Epo plus hypothermia in an HIE population, we evaluated the pharmacokinetics and safety of 5 intravenous doses of Epo administered between 1 and 9 days of age (NCT No. 00719407) [11]. Of the doses tested $(250,500,1,000$, and 2,500 U/kg/dose), 1,000 U/kg/dose produced target plasma Epo levels that conferred optimal neuroprotection in animal studies [16, 18]. Neurodevelopmental outcomes were available for 22 out of 24 infants [7] at 22 months (range 8-34). There were no deaths. Moderate to severe disability occurred in only 1 child (4.5\%), despite 8 (36\%) having moderate to severe brain injury on neonatal MRI [7].

Based on the pharmacokinetic data obtained in our phase I study, we randomized 50 newborns undergoing hypothermia for moderate/severe HIE to 5 intravenous doses of Epo $(1,000 \mathrm{U} / \mathrm{kg} /$ dose) or placebo (NCT No. 01913340). Infants received the study drug on days 1,2 ,
3, 5, and 7 of age. The 24 infants who received Epo exhibited less brain injury on MRI, and better 12-month motor outcomes on the Alberta Infant Motor Scale than the 26 infants who received placebo [19]. These phase I and II data demonstrating safety and potential efficacy led us to design the phase III randomized controlled trial described herein.

\section{Study Design}

The HEAL Trial is a multicenter, randomized, doubleblind, placebo-controlled trial.

\section{Hypothesis}

Multiple doses of Epo (1,000 U/kg/dose) given to cooled infants with moderate/severe HIE will reduce the primary outcome of death or neurodevelopmental impairment (NDI) at 24 months from 49 to $33 \%$. We further hypothesize that neonatal Epo will be safe, will decrease brain injury severity on neonatal brain MRI and MRS, 
Table 2. Modified HEAL Sarnat scoring system

\begin{tabular}{|c|c|c|c|c|}
\hline 1. Level of consciousness & Hyperalert or irritable & $\begin{array}{l}\text { Lethargic or poorly } \\
\text { responsive }\end{array}$ & Minimal or no responsiveness & \\
\hline 2. Spontaneous activity & Slightly decreased & Decreased & Absent & \\
\hline 3. Posture & Mild distal flexion & $\begin{array}{l}\text { Distal flexion, complete } \\
\text { extension }\end{array}$ & Decerebrate & \\
\hline \multicolumn{5}{|l|}{ 5. Primitive reflexes } \\
\hline a. Suck & Normal & Weak or bite & Absent & Unable to assess \\
\hline b. Moro & Low threshold to elicit & Weak or incomplete & Absent & Unable to assess \\
\hline \multicolumn{5}{|l|}{ 6. Autonomic } \\
\hline a. Pupils & Equal and reactive & Constricted & $\begin{array}{l}\text { Dilated and either fixed or } \\
\text { sluggishly reactive; asymmetric }\end{array}$ & Unable to assess \\
\hline b. Respiration & Normal & Periodic breathing & Intubated and ventilated & \\
\hline
\end{tabular}

To be eligible for the HEAL study, 3 of the 6 categories must be either moderate or severe. Level of consciousness may be the deciding factor to assign the HIE stage in the event of a tie. The ideal qualifying Sarnat exam should be the worst exam that is performed prior to receiving sedating medications and between 1 and $6 \mathrm{~h}$ of age.

and will decrease serial inflammatory cytokines and biomarkers of brain injury. We expect these findings to change clinical practice.

\section{Population}

The HEAL Trial will enroll 500 newborns $\geq 36$ weeks gestational age with moderate/severe HIE determined by neurologic symptoms, Apgar score, acidosis level, and need for resuscitation. Parental consent, randomization, and initial treatment will occur within $24 \mathrm{~h}$ of birth. The inclusion and exclusion criteria are described in Table 1. Enrollment is irrespective of gender, religion, race, and ethnicity.

\section{Intervention}

Randomization is stratified by site (Appendix) and by severity of encephalopathy (Table 2). Following randomization, the study drug (Epoetin alfa, 1,000 U/kg/dose or equal volume of normal saline placebo) will be given intravenously on study days $1,2,3,4$, and 7 . Study members are blinded to the treatment group with the exception of site pharmacists and the study statistician.

\section{Neuroimaging}

A standardized 3-T brain MRI and MRS will be performed after rewarming, between 96 and $144 \mathrm{~h}$ of age. MRI and MRS data acquisition were harmonized across different MRI makes and models, using a standardized
HEAL MRI and MRS protocol through an iterative quality control process. The brain MRI studies will be read centrally by 2 independent readers blinded to the treatment arm. The severity and location of brain injury will be determined using the validated Washington University MRI scoring system specifically designed to evaluate the extent of HIE injury [19]. Quantitative MRS data will be processed using the LC Model.

\section{Plasma and Urine Biomarkers}

Each subject will have 3 blood samples (prior to study drug, and on study days 2 and 4), and 2 urine samples taken (prior to study drug, and after rewarming). Figure 1 summarizes the timing of treatment administration, collection of study specimens, and ascertainment of clinical outcomes.

\section{Outcome}

After hospital discharge, standardized parental telephone interviews at 4, 8, 12,18, and 24 months will collect interval medical and developmental history. The Warner Initial Developmental Evaluation A (WIDEA) parental questionnaire, which assesses development in 4 domains (self-care, mobility, communication, and social cognition), will be administered at 12, 18, and 24 months [20].

The primary composite outcome of death or mild/ moderate/severe NDI will be assessed at 24 months of age. Impairment is defined as any of the following: Gross 

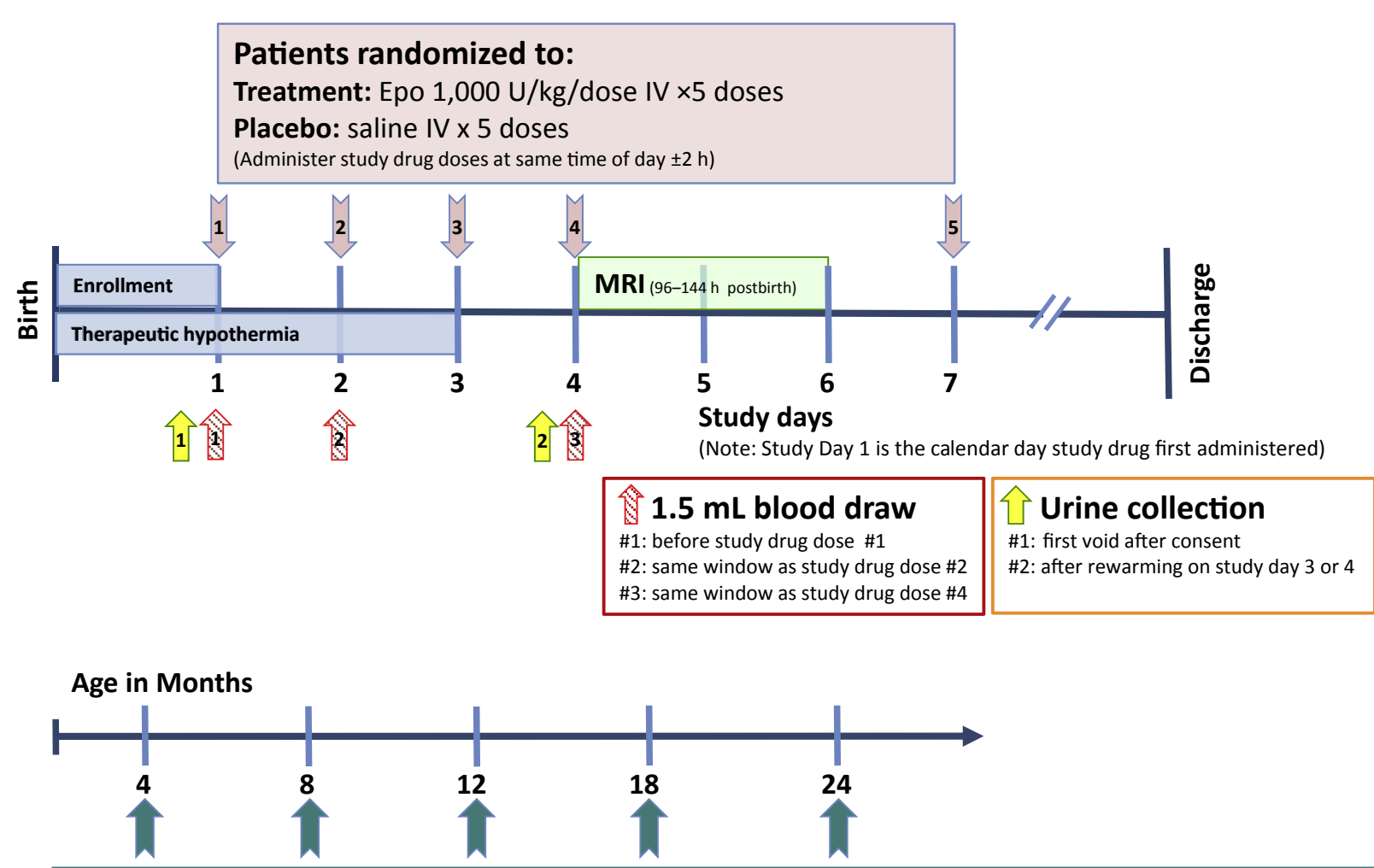

Phone follow-up (4, 8, 12, 18, and 24 months) In-clinic follow-up (24 months)

Interval medical and social history

WIDEA $(12,18$, and 24 months)

Bayley III

Standardized neurologic exam

Gross Motor Function Classification System (GMFCS)

Child Behavior Checklist (CBCL)

Fig. 1. Study protocol summary.

Motor Function Classification System (GMFCS) level $\geq 1$, or GMFCS $=0$ or 0.5 and CP (any type), or Bayley Scales of Infant and Toddler Development 3rd Edition (Bayley III) cognitive score $<90$.

$\mathrm{CP}$ presence and type will be determined by a validated standardized neurologic examination [21]. All neurologic and Bayley III examiners will be centrally trained and certified. Since death is a competing outcome with NDI, it is critical to include it in the primary outcome measure.

We will assess the effect of Epo on secondary outcomes at 24 months of age, including: (a) the presence of CP, (b) severity of motor impairment based on the GMFCS score, (c) Bayley III cognitive and language scores, (d) epilepsy (i.e., $\geq 2$ afebrile, unprovoked seizures), and (e) behavioral abnormalities (i.e., attention problems or aggressive behavior) based on the Child Behavior Checklist externalizing score.

To evaluate whether Epo shifts the overall severity of impairment, we will perform a secondary analysis of the effect of Epo on a 4-level outcome: (1) normal, (2) mild motor and/or cognitive impairment, (3) moderate/severe motor and/or cognitive impairment, and (4) death. The severity of motor impairment will be determined by the type of CP and GMFCS level. The severity of cognitive impairment will be determined by the Bayley III cognitive score.

\section{Safety}

Complications of HIE and potentially of the study drug are captured through standardized case report forms (CRFs). Prespecified serious adverse events (SAEs) are 
Table 3. Prespecified SAEs

Systemic hypertension requiring anti-hypertensive therapy

Polycythemia

Disseminated intravascular coagulation (clinical bleeding/oozing requiring transfusion of blood product)

Major venous or arterial thrombosis not related to a central line

Pulmonary hypertension treated with inhaled nitric oxide or extracorporeal life support

Intracranial hemorrhage visualized on head ultrasound or MRI T1 or T2 sequences

Cardiopulmonary arrest not secondary to ETT obstruction or other mechanical issue

Death

documented and adjudicated by an independent medical monitor masked to treatment allocation (Table 3).

All CRF data are remotely reviewed for data quality and completeness. On-site monitoring visits ensure protocol and regulatory compliance. An NIH-led Data Safety Monitoring Board (DSMB) meets regularly to oversee study conduct and safety. At prespecified interim enrollment time points of $n=125,250$, and 375 subjects, the DSMB will formally compare mortality and SAE rates across the 2 treatment groups using appropriate small sample methods such as the Fisher exact test. The DSMB will monitor mortality as a primary safety endpoint. The overall significance level will be controlled using O'BrienFleming boundaries [22]. An interim analysis of efficacy/ futility will not be conducted, as at the time the primary measure of treatment efficacy can be assessed, the majority of patients will have already been randomized.

\section{Sample Size}

Using data from 3 large sites in the phase II study [19], we estimated the following outcomes: death, $14 \%$; moderate/severe impairment, $18 \%$; mild impairment, $17 \%$; normal, $51 \%$. Therefore, we anticipate the rate of death or $\mathrm{NDI}$, the primary outcome, to be $49 \%$ in placebo-treated controls. The primary outcome will be assessed between 22 and 26 months of age. We considered data from a longterm follow-up phase I study [11] in which 24 cooled infants were given multiple doses of Epo ranging from 250 to $2,500 \mathrm{U} / \mathrm{kg}$ [7]. At $22-26$ months of age, a total of 22 subjects were followed. Of these, 0 subjects died, 1 had moderate-severe NDI, and 6 had mild NDI (overall primary outcome rate of $7 / 22=31.8 \%$; exact confidence interval $=14-55 \%$ for our planned intervention group). Based on animal data [23], phase I data [11], and projections from phase II data [19], we expect primary outcome rates ranging from 31 to $35 \%$ among Epo-treated subjects with a protective relative risk of $0.65-0.71$. An enrolled sample of 500 subjects, and assuming an intervention rate of $33 \%$, yields greater than $90 \%$ power to detect a difference from a control rate of $49 \%$ (relative risk $=0.67$ ). We assume a $90 \%$ follow-up rate with $n=225 / 250$ subjects evaluated in each arm.

\section{Statistical Analyses}

Analyses will be based on a modified intention to treat (mITT) approach. All randomized neonates receiving at least 1 study drug dose will be analyzed. Neonates withdrawn after randomization but before the first dose will be excluded from the mITT. The primary analysis will test equality of the primary outcome (death or NDI) rate across the 2 groups using a likelihood ratio test based on logistic regression, with stratification by site and HIE severity. For the ordered categorical secondary long-term outcome (death; moderate/severe impairment; mild impairment; normal), we will use a generalization of the Wilcoxon test that controls for site and HIE severity.

We will select a random subset of 200 subjects (100 treated and 100 controls, including both moderate and severe HIE) to measure circulating biomarkers of inflammation and brain injury. Time-specific comparisons of the mean biomarker will be measured across treatment groups using appropriate regression methods while controlling for site and HIE severity. In addition, we will conduct a longitudinal analysis using linear mixed models [24] that permit an omnibus test across all 3 blood sample measurement times, and allow inference on differential rates of change across treatment groups. For the quantitative MRI-based injury score (Washington University MRI scoring system), we will use a stratified $t$ test to provide inference regarding the mean response across the treatment groups adjusting for site and HIE severity.

\section{Limitations}

The majority of HEAL subjects are transferred from referring institutions, challenging the time-sensitive consent process. Successful enrollments require timely coor- 
dination amongst transport, neonatology, and neurology teams at referring and receiving hospitals. Furthermore, the Sarnat exam (on which randomization is based) must be performed consistently across sites, and within 1-6 h of life. To facilitate consistent Sarnat exams, we created an online training and examiner certification process. Harmonization of MR studies across platforms is also challenging, and the timing of the MR exams cannot always be done within the ideal window due to clinical complications.

\section{Discussion}

This research has the potential to reduce suffering from life-long neurologic disabilities, and to significantly reduce the societal costs of caring for survivors with NDI. In 2012 currency, the lifetime cost of caring for an individual with $\mathrm{CP}$ is estimated at USD 1.15 million [25]. Using a conservative estimate of $20 \%$ CP rates in infants with HIE treated with hypothermia and a conservative HIE incidence of 2 per 1,000 , each year infants born with HIE introduce an economic burden that will total USD 1.7 billion in lifetime costs due to CP alone. Similar calculations using CDC cost data [25], and rates of disability derived from hypothermia studies suggest that each year HIE produces additional lifetime costs of USD 1.6 billion for intellectual disability. While assessments at 2 years of age are critical, ultimately, longerterm follow-up will be necessary to determine whether combined Epo and hypothermia treatment results in sustained improved long-term outcomes such as school readiness, IQ, executive function, and motor function. If no benefits of Epo are found, the HEAL Trial will nevertheless generate important information about clinical and laboratory biomarkers of neurodevelopmental disabilities related to newborn encephalopathy, and the data will inform the next generation of neonatal neuroprotection trials.

\section{Acknowledgements}

Clinical Coordinating Center

Yvonne Wu, Sandra Juul, Dennis Mayock, Fernando Gonzalez, Amy Goodman, Stephanie Hauge, Samantha Nikirk, Kelleen Nelson.

Data Coordinating Center

Patrick Heagerty, Bryan Comstock, Christopher Nefcy, Mark Konodi.
Neuroimaging Committee

Robert McKinstry, Jessica Wisnowski, Stefan Bluml, Ashok Panigrahy, Amit Mathur, Yvonne Wu, Sandra Juul, Kelleen Nelson.

Follow-Up Committee

Elizabeth Rogers, Jean Lowe, Karl Kuban, Mike O’Shea, Yvonne $\mathrm{Wu}$, Sandra Juul.

Data Safety and Monitoring Committee

Ronnie Guillet (DSMB chair), Robin Ohls, Janet Soul, Jody Ciolino, Renee Shellhaas.

Independent Medical Monitor

Mike Schreiber.

\section{Disclosure Statement}

The authors have no conflicts of interest.

\section{Funding Sources}

NINDS 1U01NS092764 and U01NS092553.

\section{Appendix}

\begin{tabular}{ll}
\hline Site & Site PI \\
\hline Cincinnati, OH & Brenda Poindexter \\
Minneapolis, MN & Ellen Bendel-Stenzel \\
St. Paul, MN & Andrea Lampland \\
Philadelphia, PA & John Flibotte \\
Los Angeles, CA & Tai-We Wu \\
Washington, DC & Taeun Chang \\
Dallas, TX & Lina Chalak \\
Fort Worth, TX & David Riley \\
Indianapolis, IN & Ulrike Mietzsch \\
Miami, FL & Magaly Diaz-Barbosa \\
Columbus, OH & Nathalie Maitre \\
Pittsburgh, PA & Toby Yanowitz \\
Stanford, CA & Krisa Van Meurs \\
San Antonio, TX & Kaashif Ahmad \\
San Francisco, CA & Fernando Gonzalez \\
Seattle, WA & Dennis Mayock \\
Nashville, TN & Hendrik Weitkamp \\
St. Louis, MO & Amit Mathur \\
\hline
\end{tabular}




\section{References}

1 Liu L, Oza S, Hogan D, Perin J, Rudan I, Lawn JE, Cousens S, Mathers C, Black RE: Global, regional, and national causes of child mortality in 2000-13, with projections to inform post-2015 priorities: an updated systematic analysis. Lancet 2015;385:430-440.

2 Pauliah SS, Shankaran S, Wade A, Cady EB, Thayyil S: Therapeutic hypothermia for neonatal encephalopathy in low- and middle-income countries: a systematic review and meta-analysis. PLoS One 2013;8:e58834.

3 Tagin MA, Woolcott CG, Vincer MJ, Whyte RK, Stinson DA: Hypothermia for neonatal hypoxic ischemic encephalopathy: an updated systematic review and meta-analysis. Arch Pediatr Adolesc Med 2012;166:558-566.

4 Rangarajan V, Juul SE: Erythropoietin: Emerging role of erythropoietin in neonatal neuroprotection. Pediatr Neurol 2014;51: 481-488.

5 Juul SE, Pet GC: Erythropoietin and neonatal neuroprotection. Clin Perinatol 2015;42:469481.

6 Deep Garg B, Sharma D, Bansal A: Systematic review seeking erythropoietin role for neuroprotection in neonates with hypoxic ischemic encephalopathy: presently where do we stand. J Matern Fetal Neonatal Med 2017, Epub ahead of print.

7 Rogers EE, Bonifacio SL, Glass HC, Juul SE, Chang T, Mayock DE, Durand DJ, Song D, Barkovich AJ, Ballard RA, Wu YW: Erythropoietin and hypothermia for hypoxic-ischemic encephalopathy. Pediatr Neurol 2014; 51:657-662.

8 Malla RR, Asimi R, Teli MA, Shaheen F, Bhat MA: Erythropoietin monotherapy in perinatal asphyxia with moderate to severe encephalopathy: a randomized placebo-controlled trial. J Perinatol 2017;37:596-601.
9 Juul S: Erythropoietin in anemia of prematurity. J Matern Fetal Neona 2012;25:80-84.

10 Frymoyer A, Juul SE, Massaro AN, Bammler TK, Wu YW: High-dose erythropoietin population pharmacokinetics in neonates with hypoxic-ischemic encephalopathy receiving hypothermia. Pediatr Res 2017;81:865-872.

11 Wu YW, Bauer LA, Ballard RA, Ferriero DM, Glidden DV, Mayock DE, Chang T, Durand DJ, Song D, Bonifacio SL, Gonzalez FF, Glass HC, Juul SE: Erythropoietin for neuroprotection in neonatal encephalopathy: safety and pharmacokinetics. Pediatrics 2012;130:683691.

12 McAdams RM, McPherson RJ, Kapur RP, Juul SE: Focal brain injury associated with a model of severe hypoxic-ischemic encephalopathy in nonhuman primates. Dev Neurosci 2017;39:107-123.

13 Hagberg H, Mallard C, Rousset CI, Thornton C: Mitochondria: hub of injury responses in the developing brain. Lancet Neurol 2014;13: 217-232.

14 Northington FJ, Ferriero DM, Flock DL, Martin LJ: Delayed neurodegeneration in neonatal rat thalamus after hypoxia- ischemia is apoptosis. J Neurosci 2001;21:1931-1938.

15 Jantzie LL, Miller RH, Robinson S: Erythropoietin signaling promotes oligodendrocyte development following prenatal systemic hypoxic-ischemic brain injury. Pediatr Res 2013;74:658-667.

16 Kellert BA, McPherson RJ, Juul SE: A comparison of high-dose recombinant erythropoietin treatment regimens in brain-injured neonatal rats. Pediatr Res 2007;61:451-455.

17 Larpthaveesarp A, Georgevits M, Ferriero DM, Gonzalez FF: Delayed erythropoietin therapy improves histological and behavioral outcomes after transient neonatal stroke. Neurobiol Dis 2016;93:57-63.
18 Statler PA, McPherson RJ, Bauer LA, Kellert BA, Juul SE: Pharmacokinetics of high-dose recombinant erythropoietin in plasma and brain of neonatal rats. Pediatr Res 2007;61: 671-675.

19 Wu YW, Mathur AM, Chang T, McKinstry RC, Mulkey SB, Mayock DE, Van Meurs KP, Rogers EE, Gonzalez FF, Comstock BA, Juul SE, Msall ME, Bonifacio SL, Glass HC, Massaro AN, Dong L, Tan KW, Heagerty PJ, Ballard RA: High-dose erythropoietin and hypothermia for hypoxic-ischemic encephalopathy: a phase II trial. Pediatrics 2016;137: e20160191.

20 Msall ME: Measuring functional skills in preschool children at risk for neurodevelopmental disabilities. Ment Retard Dev Disabil Res Rev 2005;11:263-273.

21 Kuban KC, Allred EN, O'Shea M, Paneth N, Pagano M, Leviton A: An algorithm for identifying and classifying cerebral palsy in young children. J Pediatr 2008;153:466-472.

22 O'Brien PC, Fleming TR: A multiple testing procedure for clinical trials. Biometrics 1979; 35:549-556.

23 Traudt CM, McPherson RJ, Bauer LA, Richards TL, Burbacher TM, McAdams RM, Juul SE: Concurrent erythropoietin and hypothermia treatment improve outcomes in a term nonhuman primate model of perinatal asphyxia. Dev Neurosci 2013;35:491-503.

24 Diggle PJ, Heagerty PJ, Liang KY, Zeger SL: Analysis of Longitudinal Data, ed 2. Oxford, Oxford University Press, 2002.

25 Centers for Disease Control and Prevention: Economic costs associated with mental retardation, cerebral palsy, hearing loss, and vision impairment - United States, 2003. Morb Mortal Wkly Rep 2004;53:57-59. 